\title{
On the Decay of Solutions for a Nonlinear Petrovsky Equation
}

\author{
Erhan Piskin* and Necat Polat \\ Department of Mathematics, Dicle University, 21280, Diyarbakir, Turkey
}

Received: 12 Jun. 2013, Revised: 8 Oct. 2013, Accepted: 9 Oct. 2013

Published online: 1 Jan. 2014

\begin{abstract}
This work studies the initial boundary value problem for the Petrovsky equation $u_{t t}+\Delta^{2} u-\Delta u_{t}+\left|u_{t}\right|^{m-1} u_{t}=|u|^{p-1} u$. Under suitable conditions decay estimates of the solution are proved by using Nakaos inequality.
\end{abstract}

Keywords: Decay, Global Existence, Petrovsky Equation

\section{Introduction}

In this work we study the following initial-boundary value problem

$\left\{\begin{array}{cc}u_{t t}+\triangle^{2} u-\triangle u_{t}+\left|u_{t}\right|^{m-1} u_{t}=|u|^{p-1} u, & (x, t) \in \Omega \times(0, T), \\ u(x, 0)=u_{0}(x), u_{t}(x, 0)=u_{1}(x), & x \in \Omega, \\ u(x, t)=\partial_{v} u(x, t)=0, & x \in \partial \Omega,\end{array}\right.$

where $\Omega$ is a bounded domain with smooth boundary $\partial \Omega$ in $R^{n}, n \geq 1 ; v$ is the outer normal.

In the absence of the strong damping term $\triangle u_{t}$, the interaction between the nonlinear damping and source term were established by many authors $[1,4]$. Recently, Li et. al [5] investigated problem (1) and showed the global existence, energy decay and blow up of the solution.

In this paper, we analyze the influence of the damping terms and source terms on the solutions of problem (1). We obtained the global existence result by potential well method. The exponential decay, for $m=1$ and the polynomial decay, for $m>1$ were established by using Nakao's inequality.

This paper is organized as follows. In section 2, we present some lemmas, and the local existence theorem. In section 3, the global existence and the decay of the solution are given.

\section{Preliminaries}

In this section, we shall give some assumptions and lemmas which will be used throughout this paper. Let \|.\| and $\|\cdot\|_{p}$ denote the usual $L^{2}(\Omega)$ norm and $L^{p}(\Omega)$ norm, respectively.

Lemma 1.(Sobolev-Poincare inequality) [2]. If $2 \leq p \leq$ $\frac{2 n}{n-4}(2 \leq p<\infty$ if $n=1,2,3,4)$, then

$$
\|u\|_{p} \leq C_{*}\|\triangle u\| \text { for } u \in H_{0}^{2}(\Omega)
$$

holds with some constant $C_{*}$.

Lemma 2.[3]. Let $\phi(t)$ be nonincreasing and nonnegative function defined on $[0, T], T>1$, satisfying

$$
\phi^{1+\alpha}(t) \leq w_{0}(\phi(t)-\phi(t+1)), t \in[0, T]
$$

for $w_{0}$ is a positive constant and $\alpha$ is a nonnegative constant. Then we have, for each $t \in[0, T]$,

$$
\left\{\begin{array}{cc}
\phi(t) \leq \phi(0) e^{-w_{1}[t-1]^{+}}, & \alpha=0, \\
\phi(t) \leq\left(\phi(0)^{-\alpha}+w_{0}^{-1} \alpha[t-1]^{+}\right)^{-\frac{1}{\alpha}}, & \alpha>0,
\end{array}\right.
$$

where $[t-1]^{+}=\max \{t-1,0\}$, and $w_{1}=\ln \left(\frac{w_{0}}{w_{0}-1}\right)$.

Next, we state the local existence theorem which is proved in [1].

Theorem 1.(Local existence). Suppose that $m$, p satisfies

$$
\begin{aligned}
& \left\{\begin{array}{c}
1<m<\infty, n \leq 4 ; \\
1<m \leq \frac{n+4}{n-4}, n>4,
\end{array}\right. \\
& \left\{\begin{array}{c}
1<p<\infty, n \leq 4 \\
1<p \leq \frac{n}{n-4}, n>4
\end{array}\right.
\end{aligned}
$$

\footnotetext{
*Corresponding author e-mail: episkin@dicle.edu.tr
} 
and further $u_{0} \in H_{0}^{2}(\Omega)$ and $u_{1} \in L^{2}(\Omega)$ such that problem (1) has a unique local solution

$u \in C\left([0, T) ; H_{0}^{2}(\Omega)\right)$ and

$u_{t} \in C\left([0, T) ; L^{2}(\Omega)\right) \cap L^{m+1}(\Omega \times[0, T))$.

Moreover, at least one of the following statements holds true:
i) $T=\infty$,
ii) $\left\|u_{t}\right\|^{2}+\|\Delta u\|^{2} \longrightarrow \infty$ as $t \longrightarrow T^{-}$.

\section{Global existence and decay of solutions}

In this section, we discuss the global existence and decay of the solution for problem (1).

We define

$$
J(t)=\frac{1}{2}\|\triangle u\|^{2}-\frac{1}{p+1}\|u\|_{p+1}^{p+1},
$$

and

$$
I(t)=\|\Delta u\|^{2}-\|u\|_{p+1}^{p+1} .
$$

We also define the energy function as follows

$$
E(t)=\frac{1}{2}\left\|u_{t}\right\|^{2}+\frac{1}{2}\|\Delta u\|^{2}-\frac{1}{p+1}\|u\|_{p+1}^{p+1} .
$$

Finally, we define

$$
W=\left\{u: u \in H_{0}^{2}(\Omega), I(u)>0\right\} \cup\{0\} .
$$

The next lemma shows that our energy functional (6) is a nonincreasing function along the solution of (1).

Lemma 3. $E(t)$ is a nonincreasing function for $t \geq 0$ and

$$
E^{\prime}(t)=-\left(\left\|u_{t}\right\|_{m+1}^{m+1}+\left\|\nabla u_{t}\right\|^{2}\right) \leq 0 .
$$

Proof.Multiplying the equation of (1) by $u_{t}$ and integrating over $\Omega$, using integrating by parts and summing up the product results, we get

$$
E(t)-E(0)=-\int_{0}^{t}\left(\left\|u_{\tau}\right\|_{m+1}^{m+1}+\left\|\nabla u_{\tau}\right\|^{2}\right) d \tau \text { for } t \geq 0 .
$$

Lemma 4.Suppose that (2) holds. Let $u_{0} \in W$ and $u_{1} \in$ $H_{0}^{m}(\Omega)$ such that

$$
\beta=C_{*}\left(\frac{2(p+1)}{p-1} E(0)\right)^{\frac{p-1}{2}}<1,
$$

then $u \in W$ for each $t \geq 0$.
Proof. Since $I(0)>0$, it follows the continuity of $u(t)$ that

$$
I(t)>0,
$$

for some interval near $t=0$. Let $T_{m}>0$ be a maximal time, when (5) holds on $\left[0, T_{m}\right]$.

From (4) and (3), we have

$$
\begin{aligned}
J(t) & =\frac{1}{p+1} I(t)+\frac{p-1}{2(p+1)}\|\Delta u\|^{2} \\
& \geq \frac{p-1}{2(p+1)}\|\triangle u\|^{2}
\end{aligned}
$$

By using (11), (6) and Lemma 3, we get

$$
\begin{aligned}
\|\Delta u\|^{2} & \leq \frac{2(p+1)}{p-1} J(t) \\
& \leq \frac{2(p+1)}{p-1} E(t) \\
& \leq \frac{2(p+1)}{p-1} E(0) .
\end{aligned}
$$

By recalling Lemma 1 and (12), we have

$$
\begin{aligned}
\|u\|_{p+1}^{p+1} & \leq C_{*}\|\Delta u\|^{p+1} \\
& =C_{*}\|\Delta u\|^{p-1}\|\triangle u\|^{2} \\
& \leq C_{*}\left(\frac{2(p+1)}{p-1} E(0)\right)^{\frac{p-1}{2}}\|\Delta u\|^{2} \\
& =\beta\|\triangle u\|^{2} \\
& <\|\triangle u\|^{2} \text { on } t \in\left[0, T_{m}\right] .
\end{aligned}
$$

Therefore, by using (5), we conclude that $I(t)>0$ for all $t \in\left[0, T_{m}\right]$. By repeating the procedure, $T_{m}$ is extended to $T$. The proof of Lemma 4 is completed.

Lemma 5.Let assumptions of Lemma 4 holds. Then there exists $\eta_{1}=1-\beta$ such that

$$
\|u\|_{p+1}^{p+1} \leq\left(1-\eta_{1}\right)\|\triangle u\|^{2} .
$$

Proof.From (13), we get

$$
\|u\|_{p+1}^{p+1} \leq \beta\|\triangle u\|^{2} .
$$

Let $\eta_{1}=1-\beta$, then we have the result.

Remark.From Lemma 5, we can deduce that

$$
\|\triangle u\|^{2} \leq \frac{1}{\eta_{1}} I(t)
$$

Theorem 2.Suppose that (2) holds. Let $u_{0} \in W$ satisfying (10). Then the solution of problem (1) is global.

Proof.It is sufficient to show that $\left\|u_{t}\right\|^{2}+\|\Delta u\|^{2}$ is bounded independently of $t$. To achieve this we use (5) 
and (6) to obtain

$$
\begin{aligned}
E(0) & \geq E(t)=\frac{1}{2}\left\|u_{t}\right\|^{2}+\frac{1}{2}\|\Delta u\|^{2}-\frac{1}{p+1}\|u\|_{p+1}^{p+1} \\
& =\frac{1}{2}\left\|u_{t}\right\|^{2}+\frac{p-1}{2(p+1)}\|\Delta u\|^{2}+\frac{1}{p+1} I(t) \\
& \geq \frac{1}{2}\left\|u_{t}\right\|^{2}+\frac{p-1}{2(p+1)}\|\triangle u\|^{2}
\end{aligned}
$$

since $I(t) \geq 0$. Therefore

$$
\left\|u_{t}\right\|^{2}+\|\triangle u\|^{2} \leq C E(0)
$$

where $C=\max \left\{2, \frac{2(p+1)}{p-1}\right\}$. Then by Theorem 1, we have the global existence result.

Theorem 3.Suppose that (2) and (10) holds, and further $u_{0} \in W$. Thus, we have following decay estimates:

$$
E(t) \leq\left\{\begin{array}{cc}
E(0) e^{-w_{1}[t-1]^{+}}, & \text {if } m=1, \\
\left(E(0)^{-\alpha}+C_{7}^{-1} \alpha[t-1]^{+}\right)^{-\frac{1}{\alpha}}, & \text { if } m>1,
\end{array}\right.
$$

where $w_{1}, \alpha$ and $C_{7}$ are positive constants which will be defined later.

Proof.By integrating (8) over $[t, t+1], t>0$, we have

$$
E(t)-E(t+1)=D^{m+1}(t),
$$

where

$$
D^{m+1}(t)=\int_{t}^{t+1}\left(\left\|u_{\tau}\right\|_{m+1}^{m+1}+\left\|\nabla u_{\tau}\right\|^{2}\right) d \tau .
$$

By virtue of (16) and Hölder inequality, we observe that

$$
\int_{t}^{t+1} \int_{\Omega}\left|u_{t}\right|^{2} d x d t \leq|\Omega|^{\frac{r+1}{r+2}} D^{2}(t)=C D^{2}(t) .
$$

Hence, from (17), there exist $t_{1} \in\left[t, t+\frac{1}{4}\right]$ and $t_{2} \in\left[t+\frac{3}{4}, t+1\right]$ such that

$$
\left\|u_{t}\left(t_{i}\right)\right\| \leq C D(t), i=1,2
$$

Multiplying the equation of (1) by $u$, and integrating it over $\Omega \times\left[t_{1}, t_{2}\right]$, we get

$$
\begin{aligned}
\int_{t_{1}}^{t_{2}} I(t) d t= & -\int_{t_{1}}^{t_{2}} \int_{\Omega} u u_{t t} d x d t-\int_{t_{1}}^{t_{2}} \int_{\Omega} \nabla u_{t} \nabla u d x d t \\
& -\int_{t_{1}}^{t_{2}} \int_{\Omega}\left|u_{t}\right|^{m-1} u_{t} u d x d t .
\end{aligned}
$$

By using (1) and integrating by parts and Cauchy-Schwarz inequality in the first term, and Hölder inequality in the second term of the right hand side of (19), we obtain

$$
\begin{aligned}
\int_{t_{1}}^{t_{2}} I(t) d t \leq & \left\|u_{t}\left(t_{1}\right)\right\|\left\|u\left(t_{1}\right)\right\|+\left\|u_{t}\left(t_{2}\right)\right\|\left\|u\left(t_{2}\right)\right\| \\
& +\int_{t_{1}}^{t_{2}}\left\|u_{t}(t)\right\|^{2} d t+\int_{t_{1}}^{t_{2}}\left\|\nabla u_{t}\right\|\|\nabla u\| d t \\
& -\int_{t_{1}}^{t_{2}} \int_{\Omega}\left|u_{t}\right|^{m-1} u_{t} u d x d t
\end{aligned}
$$

Now, our goal is to estimate the last term in the right-hand side of inequality (20). By using Hölder inequality, we obtain

$$
\int_{t_{1}}^{t_{2}} \int_{\Omega}\left|u_{t}\right|^{m-1} u_{t} u d x d t \leq \int_{t_{1}}^{t_{2}}\left\|u_{t}(t)\right\|_{m+1}^{m}\|u(t)\|_{m+1} d t
$$

By applying the Sobolev-Poincare inequality and (12), we find

$$
\begin{aligned}
& \int_{t_{1}}^{t_{2}}\left\|u_{t}(t)\right\|_{m+1}^{m}\|u(t)\|_{m+1} d t \\
\leq & C_{*} \int_{t_{1}}^{t_{2}}\left\|u_{t}(t)\right\|_{m+1}^{m}\|\Delta u(t)\| d t \\
\leq & C_{*}\left(\frac{2(p+1)}{p-1} E(0)\right)^{\frac{1}{2}} \int_{t_{1}}^{t_{2}}\left\|u_{t}(t)\right\|_{m+1}^{m} E^{\frac{1}{2}}(s) d t \\
\leq & C_{*}\left(\frac{2(p+1)}{p-1} E(0)\right)^{\frac{1}{2}} \sup _{t_{1} \leq s \leq t_{2}} E^{\frac{1}{2}}(s) \int_{t_{1}}^{t_{2}}\left\|u_{t}(t)\right\|_{m+1}^{m} d t \\
\leq & C_{*}\left(\frac{2(p+1)}{p-1} E(0)\right)^{\frac{1}{2}} \sup _{t_{1} \leq s \leq t_{2}} E^{\frac{1}{2}}(s) D^{m}(t) . \\
& \int_{t_{1}}^{t_{2}}\left\|\nabla u_{t}\right\|\|\nabla u\| d t \\
\leq & C_{*} \int_{t_{1}}^{t_{2}}\left\|\nabla u_{t}\right\|\|\Delta u(t)\| d t \\
\leq & C_{*}\left(\frac{2(p+1)}{p-1} E(0)\right)^{\frac{1}{2}} \int_{t_{1}}^{t_{2}}\left\|\nabla u_{t}\right\| E^{\frac{1}{2}}(s) d t \\
\leq & C_{*}\left(\frac{2(p+1)}{p-1} E(0)\right)^{\frac{1}{2}} \sup _{t_{1} \leq s \leq t_{2}} E^{\frac{1}{2}}(s) \int_{t_{1}}^{t_{2}}\left\|\nabla u_{t}\right\| d t,
\end{aligned}
$$

which implies

$$
\begin{aligned}
\int_{t_{1}}^{t_{2}}\left\|\nabla u_{t}\right\| d t & \leq\left(\int_{t_{1}}^{t_{2}} 1 d t\right)^{\frac{1}{2}}\left(\int_{t_{1}}^{t_{2}}\left\|\nabla u_{t}\right\|^{2} d t\right)^{\frac{1}{2}} \\
& \leq C D(t)
\end{aligned}
$$

Then

$\int_{t_{1}}^{t_{2}}\left\|\nabla u_{t}\right\|\|\nabla u\| d t \leq C C_{*}\left(\frac{2(p+1)}{p-1} E(0)\right)^{\frac{1}{2}} \sup _{t_{1} \leq s \leq t_{2}} E^{\frac{1}{2}}(s) D(t)$

From (12), (18) and Sobolev-Poincare inequality, we have

$$
\left\|u_{t}\left(t_{i}\right)\right\|\left\|u\left(t_{i}\right)\right\| \leq C_{1} D(t) \sup _{t_{1} \leq s \leq t_{2}} E^{\frac{1}{2}}(s)
$$

where $C_{1}=2 C_{*}\left(\frac{2(p+1)}{p-1} E(0)\right)^{\frac{1}{2}}$. Then by (20)-(24) we have

$$
\begin{aligned}
\int_{t_{1}}^{t_{2}} I(t) d t \leq & C_{1} \sup _{t_{1} \leq s \leq t_{2}} E^{\frac{1}{2}}(s) D(t)+D^{2}(t) \\
& +C C_{*}\left(\frac{2(p+1)}{p-1} E(0)\right)^{\frac{1}{2}} \sup _{t_{1} \leq s \leq t_{2}} E^{\frac{1}{2}}(s) D(t) \\
& +C_{*}\left(\frac{2(p+1)}{p-1} E(0)\right)^{\frac{1}{2}} \sup _{t_{1} \leq s \leq t_{2}} E^{\frac{1}{2}}(s) D^{m}(t) .
\end{aligned}
$$


On the other hand, from (5), (6) and Remark, we obtain

$$
E(t) \leq \frac{1}{2}\left\|u_{t}\right\|^{2}+C_{3} I(t),
$$

where $C_{3}=\frac{1}{\eta_{1}} \frac{p-1}{2(p+1)}+\frac{1}{p+1}$.

By integrating (26) over $\left[t_{1}, t_{2}\right]$, we have

$$
\int_{t_{1}}^{t_{2}} E(t) d t \leq \frac{1}{2} \int_{t_{1}}^{t_{2}}\left\|u_{t}\right\|^{2} d t+C_{3} \int_{t_{1}}^{t_{2}} I(t) d t .
$$

Then by (21) and (27), we get

$$
\begin{aligned}
\int_{t_{1}}^{t_{2}} E(t) d t \leq & \frac{1}{2} C D^{2}(t)+C_{3} C_{2}\left[\sup _{t_{1} \leq s \leq t_{2}} E^{\frac{1}{2}}(s) D(t)+D^{2}(t)\right. \\
& +C C_{*}\left(\frac{2(p+1)}{p-1} E(0)\right)^{\frac{1}{2}} \sup _{t_{1} \leq s \leq t_{2}} E^{\frac{1}{2}}(s) D(t) \\
& \left.+C_{*}\left(\frac{2(p+1)}{p-1} E(0)\right)^{\frac{1}{2}} \sup _{t_{1} \leq s \leq t_{2}} E^{\frac{1}{2}}(s) D^{m}(t)\right](28)
\end{aligned}
$$

By integrating $\frac{d}{d t} E(t)$ over $\left[t, t_{2}\right]$, we obtain

$$
E(t)=E\left(t_{2}\right)+\int_{t}^{t+1}\left(\left\|u_{\tau}\right\|_{m+1}^{m+1}+\left\|\nabla u_{\tau}\right\|^{2}\right) d \tau
$$

Therefore, since $t_{2}-t_{1} \geq \frac{1}{2}$, we conclude that

$$
\int_{t_{1}}^{t_{2}} E(t) d t \geq\left(t_{2}-t_{1}\right) E\left(t_{2}\right) \geq \frac{1}{2} E\left(t_{2}\right) .
$$

That is,

$$
E\left(t_{2}\right) \leq 2 \int_{t_{1}}^{t_{2}} E(t) d t
$$

Consequently, exploiting (15), (28)-(30), and since $t_{1}, t_{2} \in$ $[t, t+1]$, we get

$$
\begin{aligned}
E(t) & \leq 2 \int_{t_{1}}^{t_{2}} E(t) d t+\int_{t}^{t+1}\left(\left\|u_{\tau}\right\|_{m+1}^{m+1}+\left\|\nabla u_{\tau}\right\|^{2}\right) d \tau \\
& =2 \int_{t_{1}}^{t_{2}} E(t) d t+D^{m+1}(t)
\end{aligned}
$$

Then, by (28), we have

$$
\begin{aligned}
E(t) \leq & \left(\frac{1}{2} C+C_{3} C_{2}\right) D^{2}(t)+D^{m+1}(t) \\
& +C_{4}\left[D(t)+D^{m}(t)\right] E^{\frac{1}{2}}(t) .
\end{aligned}
$$

Hence, by Young inequality, we obtain

$$
E(t) \leq C_{5}\left[D^{2}(t)+D^{m+1}(t)+D^{2 m}(t)\right] .
$$

Case 1: When $m=1$, from (32), we obtain

$$
E(t) \leq 3 C_{5} D^{2}(t)=3 C_{5}[E(t)-E(t+1)] .
$$

By Lemma 2, we get

$$
E(t) \leq E(0) e^{-w_{1}[t-1]^{+}}
$$

where $w_{1}=\ln \frac{3 C_{5}}{3 C_{5}-1}$.

Case 2: When $m>1$, from (32), we obtain

$$
E(t) \leq C_{5} D^{2}(t)\left(1+D^{m-1}(t)+D^{2(m-1)}(t)\right) .
$$

Then since $E(t) \leq E(0), \forall t \geq 0$, we see from (15)

$$
\begin{aligned}
E(t) & \leq C_{5}\left(1+E^{\frac{m-1}{m+1}}(0)+E^{\frac{2(m-1)}{m+1}}(0)\right) D^{2}(t) \\
& \leq C_{6} D^{2}(t), \quad t \geq 0 .
\end{aligned}
$$

Then we obtain

$$
\begin{aligned}
E(t)^{\frac{m+1}{2}} & \leq C_{7} D^{m+1}(t) \\
& \leq C_{7}(E(t)-E(t+1)) .
\end{aligned}
$$

Thus, from (33) and Lemma 2, we have

$$
E(t) \leq\left(E(0)^{-\alpha}+C_{7}^{-1} \alpha[t-1]^{+}\right)^{-\frac{1}{\alpha}} .
$$

The proof of Theorem 3 is completed.

\section{References}

[1] S. A. Messaoudi, Global existence and nonexistence in a system of Petrovsky, J. Math. Anal. Appl., 265, 296-308 (2002).

[2] R. A. Adams, J. J. F. Fournier, Sobolev Spaces, Academic Press, (2003).

[3] M. Nakao, Asymptotic stability of the bounded or almost periodic solution of the wave equation with nonlinear dissipative term, J. Math. Anal. Appl., 58, 336-343 (1977).

[4] S. T. Wu, L. Y. Tsai, On global solutions and blow-up of solutions for a nonlinearly damped Petrovsky system, Taiwanese J. Math., 13, 545-558 (2009).

[5] G. Li, Y. Sun, W. Liu, Global existence and blow up of solutions for a strongly damped Petrovsky system with nonlinear damping, Appl. Anal., 91, 575-586 (2012). 


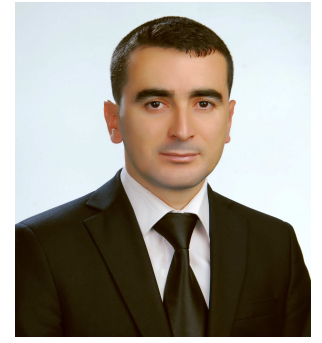

Erhan Pişkin received his $\mathrm{BS}, \mathrm{MS}$ and $\mathrm{PhD}$ degrees in Mathematics from at the Dicle University, Diyarbakr, Turkey (2005, 2009, 2013). He currently works as a research assistant at the Department of Mathematics, Dicle University, Turkey. His research interests are in local existence, global existence, continuous dependence, global nonexistence, asymptotic behavior and decay of solutions for nonlinear hyperbolic differential equations, analysis of nonlinear differential equations, and mathematical behavior of nonlinear differential equations.

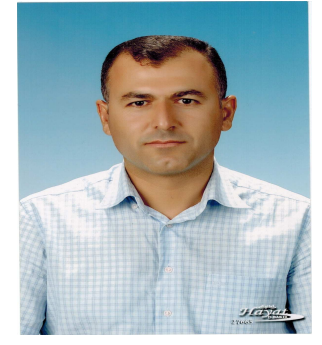

Necat

Polat graduated from Department of Mathematics, Dicle University, Diyarbakr, Turkey in 1997 and received her MS and $\mathrm{PhD}$ degrees in Applied Mathematics from Dicle University in 2000 and 2005, respectively. He currently works as an Associate Professor at Department of Mathematics in Dicle University. His research interests are in local existence, global existence, continuous dependence, global nonexistence, asymptotic behavior, decay, stability and instability of solutions for nonlinear hyperbolic and parabolic differential equations, analysis of nonlinear differential equations, and mathematical behavior of nonlinear differential equations. 